reader to further their knowledge.

The text is very readable and easy to follow with appropriate diagrams. Tables are provided to summarise data or study results as required. Clinical photographs do not feature strongly however, for the most part, this is not a subject which readily lends itself to this type of imagery.

Unfortunately, there is some repetition in the introduction to several of the chapters. This is perhaps a reflection on the number of contributors involved. This disrupts the continuity of the book somewhat. In addition, photographs of the oral appliances described would be helpful. Readers would also benefit from summary boxes interspersed among the text.

The editors fulfil their aim of producing a book which will provide easily accessible information. However, a lecture series to complement the text would be beneficial for students. This book will appeal to all interested general practitioners, orthodontists and maxillofacial surgeons. Sleep medicine physicians may also be interested to learn more about the role of the dentist in the recognition and management of sleep disorders.

S. Lucey

\section{BIOLOGICAL MECHANISMS OF TOOTH MOVEMENT}

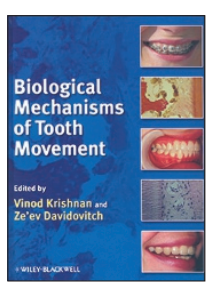

V. Krishnan, Z. Davidovitch (eds)

UK: Wiley-Blackwell price £99.50; pp 241 ISBN 9781405176903

This is an interesting and well structured book aimed at both orthodontic trainees and specialists. The text is divided into 12 chapters which detail the history of orthodontics, the biological influences on tooth movement, relapse and genetic factors affecting orthodontic tooth alignment. The effects of systemic disease, common medications and diet are also considered.

Each chapter is simply organised with an opening summary, followed by an introduction, discussion and conclusion. Detailed references and recommendations for further reading provide an important resource for those who may wish to read further into the various topics. It is well illustrated with easy to read diagrams, cephalometric tracings, graphs, tables and clinical photographs. This book provides an overview of previous research, discussing limitations within it, current concepts and the possible developments in orthodontics in the future.

An in-depth explanation of the tissue reactions upon varied force applications including mechanobiology and then mechanotransduction in bone tissue is provided. Practitioners are encouraged to remember that orthodontic tooth movement is interrelated with the systemic health of the patient and, as such, a good understanding of both is required.

The authors draw attention to the crucial function of inflammation in the remodelling process during tooth movement. The discussion involves the various biochemical mediators which are released in the periodontium. Various types of analgesia are discussed but in particular the use of non-steroidal antiinflammatory drugs.

The four page comprehensive table of the molecules associated with orthodontic tooth movement provides a comprehensive overview of the molecular events and changes in gene expression that influence tooth movement.

The biological aspects of bone growth and metabolism are explained. The authors provide a good explanation of bone development including endochondral and intramembranous bone formation. It logically proceeds to an explanation of bone remodelling involving cortical and trabecular bone which is a crucial concept in orthodontics. Attention is also directed at the optimal forces required for tooth movement, its application in common malocclusions as well as the biological basis of orthodontic relapse.

It is important to note that the discussion on antibiotic prophylaxis for infective endocarditis is based on the American Heart Association and not on the National Institute for Health and Clinical Excellence guidelines which are currently followed by practitioners in the UK.

In conclusion, this book provides a detailed, well structured and systematic overview of the biological mechanisms of tooth movement.

J. Harnett 